\title{
SILVER COLLOID STAINING OF SCLEROTIC AND NON-SCLEROTIC DENTINE
}

\author{
M. Mohd. Bakri, N.H. Mohamed, D.A. Whittaker. \\ Silver colloid staining of sclerotic and non-sclerotic \\ dentine. Annal Dent Univ Malaya 2003; 10: 11-13.
}

\begin{abstract}
Phosphophoryn, a higWy phosphorylated protein, is the most abundant protein among the non-collagenous protein of dentine. The staining of phosphophoryn can be done by using the silver colloid staining. In this paper, the staining effect of the silver colloid stain on both non-sclerotic and sclerotic dentine was investigated. Eight teeth from donors aged 14, 17, 22, $34,55,57,60$ and 65 were used for this experiment. The younger teeth were used to demonstrate normal root dentine while the older age teeth were used to demonstrate sclerotic root dentine at the apical region of the root. There was no staining of the normal root dentine as compared to sclerotic dentine when the silver colloid staining was used.
\end{abstract}

Key words: Phosphophoryn, silver colloid stain, sclerotic dentine.

\section{INTRODUCTION}

Organic matrix of dentine is comprised of collagen fibres and a variety of non-collagenous proteins. The most abundant of the non-collagenous proteins is the highly phosphorylated protein, phosphophoryn (1) which is thought to be involved in regulating the organised deposition of mineral crystals within the matrix (2). In human teeth, dentine phosphophoryn can be found in circumpulpal dentine but not in mantle dentine, reparative dentine and dentine of dentinogenesis imperfecta, a genetic disorder of dentine formation (3). Such evidence suggests that dentine phosphophoryn is synthesised and secreted only by physiologically differentiated odontoblasts but not by undifferentiated or damaged cells.

Phosphoproteins can be demonstrated histologically by silver colloid staining as it has the greatest affinity for the stain (4). Silver colloid staining was initially developed by Smith et al (5) for the detection of 'nucleolar organiser regions' (NORS) in histological sections. The numbers, size and activity of NORs have been reported to increase in transformed, proliferating or malignant cells. Whilst examining NORs activity during odontogenesis with this silver colloid stain, they observed specific reactivity with the dentine extracellular matrix in demineralised histological sections. The organic matrix of peritubular dentine was found to have the greatest

\section{Original Article}

\author{
M. Mohd. BakrP, N.H. Mohamed², \\ D.A. Whittaker ${ }^{3}$ \\ IDepartment of Oral Biology, \\ 2Unit of General Dental Practice \\ Faculty of Dentistry, University of Malaya. \\ 50603 Kuala Lumpur, Malaysia. \\ 3Department of Basic Dental Science \\ Dental School, Heath Park, Cardiff CF4 4XY, \\ University of Wales College of Medicine \\ United Kingdom. \\ Corresponding author - Marina Mohd. Bakri
}

affinity for the stain.

Phosphoprotein, when purified, has been found to contain a large proportion of aspartic acid residues and with age, some of them were inverted from the Lisomer to the D-isomer (6). In dentine, the D-aspartic acid increases with age $(6,7,8)$ and quantification of the D-aspartic acid as in the racemization method has shown to be very accurate when used to estimate age (9). Apart from aspartic acid, the serine content of phosphoprotein has been shown to decrease dramatically with age (10). Therefore, it may be concluded that phosphoprotein has played a role in forensic investigation of estimating age.

The use of sclerotic dentine in age estimation is also well established and due to its high mineral content, the amount of phosphoprotein would probably be lesser than normal dentine. In this paper, the staining effect of the silver colloid stain on both normal and sclerotic dentine was investigated.

\section{MATERIALS AND METHOD}

Selection of specimen:

Eight teeth from donors aged 14, 17,22, 34, 55, 57, 60 and 65 years were used for this experiment. The teeth collected for this study had minimal caries or were caries free. The younger teeth were chosen to demonstrate normal root dentine as the amount of sclerotic dentine if present is minimal. In order to demonstrate sclerotic dentine, older aged teeth were chosen where the distribution of the sclerotic dentine at the apical root would be obvious.

Preparation of specimen:

Teeth were fixed in $10 \%$ normal saline for one day before demineralisation by $10 \%$ formic acid. The end point of the demineralisation was determined by radiography of the specimen. Teeth were then 
processed through graded alcohols, xylene and finally embedded in paraffin wax. Processed teeth were embedded in fresh paraffin wax in Tissue Tek cassettes and moulds. With the use of the microtome, $3 / \mathrm{Lm}$ sections were obtained from paraffin wax embedded blocks of each tooth. The sections were dewaxed in xylene for 5 minutes and hydrated through 100\% ethanol to deionised water and washed thoroughly for 10 minutes.

\section{Preparation of staining solution:}

Powdered gelatine was dissolved in deionised water in a water bath at a concentration of $2 \%$. Pure formic acid was added to the solution to give it a final concentration of $1 \%$. An aqueous solution of $60 \%$ silver nitrate was prepared. The former mixture was added to the staining preparation at a volume ratio of $2: 1$. The mixed solution was then filtered through a 20/Lm Milipore filter and kept in the dark.

Sections were placed in the working solution in coplin jars placed in a dark place at ambient room temperature for 45 minutes. They were washed in deionised water for 10 minutes and dehydrated in $100 \%$ ethanol. The sections were then cleared in xylene before they were mounted in synthetic resin for microscopic viewing.

\section{RESULTS}

Following silver colloid staining, normal root dentine as observed in the younger aged teeth as well in the coronal aspects of the older aged teeth, stained deep golden brown (Fig 1). The sclerotic dentine of the apical root region of the older aged teeth was not stained by the silver colloid. Background precipitation of the silver was observed in the histological sections but this was insufficient to mask the staining characteristics (Fig 2). Although teeth chosen for this method had minimal caries, it was noted that in areas where caries had occurred, the sclerosed dentinal tubules associated with dentinal reaction to caries, stained poorly or negatively with silver colloid.

\section{DISCUSSION}

The difference in the staining properties of normal and sclerotic dentine root dentine when subjected to silver colloid appears to suggest a difference in the biochemical properties of the two types of dentine. This is likely to be associated with the non-collagenous matrix component. Silver colloid stain is known to have a high affinity for the non-collagenous component of peritubular dentine of which the phosphoprotein component is the most abundant (1). In this present study, the normal root dentine stained deep golden brown (Figure 1) and this has also been reported by Smith et al (5) while in the zone of sclerosis, there

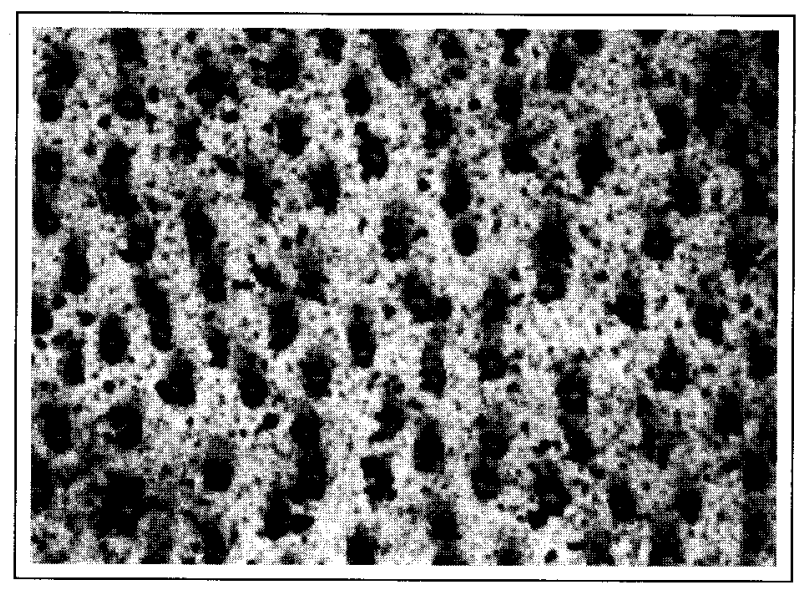

Figure 1: Normal root dentine stained with silver colloid.

Phosphoprotein, the most abundant component of the non-collagenous protein can be demonstrated histologically by the silver colloid stain as seen in the peritubular dentine. X 400 .

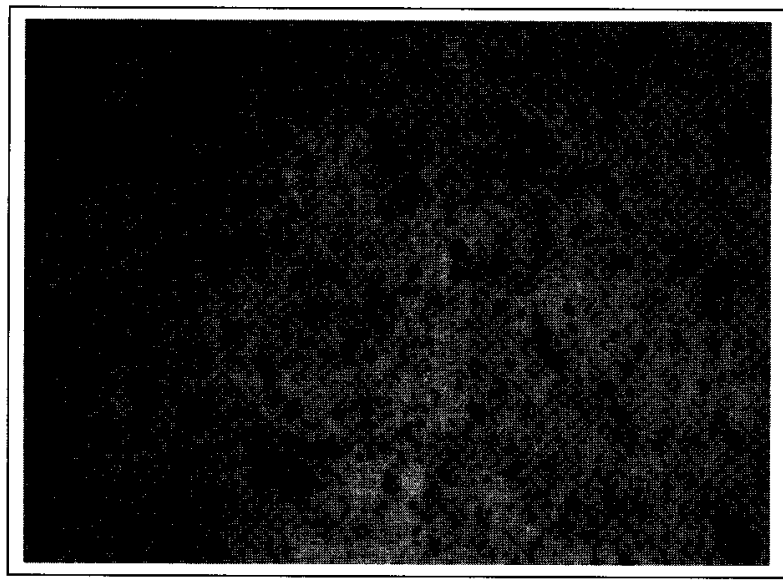

Figure 2: Staining of sclerotic dentine with silver colloid. No staining of the peritubular dentine can be seen and this may be due to the reduced non-collagenous component of the sclerotic dentine. X 400 .

was no staining of the sclerosed dentine by the silver colloid (Figure 2).

Using biochemical analyses, Takagi et al (3) found that root dentine contained only half the amount of dentine phosphophoryn present in crown dentine. Further work was carried out using a histochemical stain, Toluidine blue, and it was found that in both crown and root, more intense staining was observed in the inner layers of dentine. The dentine near the pre-dentine border was stained intensely while mantle dentine, the outermost layer of dentine was barely stained. Differences in the staining density of crown and root dentine confirmed that the amount of the phosphophoryn of the root dentine was also less than that of the crown.

If root dentine contained only half the amount of the non-collagenous component of crown dentine, this could mean that the non-collagenous component 
present in the apical sclerotic dentine would be even less than the amount present in normal root dentine. This is because with age, when the process of sclerosis is in progress, the proportion of non-collagenous component would be reduced due to the increased mineralization occurring with age. As it is this component that has the highest affinity for the silver colloid stain, it is to be expected that the peritubular dentine of the sclerotic region would loose its affinity for the stain.

When sclerotic areas of older aged teeth examined, there was no specific staining except for a background precipitation of silver which was present in all sections. In the coronal portions of the teeth, sclerosis associated with attrition or early dentinal caries stained rather poorly suggesting that the biochemical properties in the sclerotic region of ageing root dentine might be similar in some aspects to those in zones of reactive dentine in the crown of teeth. In conclusion, the differences in the staining properties of normal and sclerotic dentine seem to suggest that there is a difference in the biochemical properties between the two types pf dentine.

\section{REFERENCES}

1. DiMuzio MT and Veis A. The biosynthesis of phosphophoryns and dentine collagen in the continuously erupting rat incisors. Journal of BioI Chern 1978; 253(19): 6845-52.

2. Butler WT. Dentine exracellular matrix and dentinogenesis. Oper Dent 1992: Suppl (5): 1823.

3. Takagi $Y$, Nagai $H$ and Sasaki S. Difference in non-collagenous matrix composition between crown and root dentine of bovine incisor. Calcif Tissue Int 1988; 42: 97-103.
4. Aulak KS, Smith AJ, Matthews JB, Potts AJC, Perry $\mathrm{H}$ and Wilson C. Phosphoproteins of dentine extracellular matrix; silver colloid staining. Bioch Soc Trans 1991; 19: 188S.

5. Smith PJ, Skilbeck N, Harrison A and Crocker J. J Pathol1988; 155: 109-12.

6. Masuda W, Nouso C, Kitamura C, Terashita M and Noguci T. D-Aspartic acid in bovine dentine non-collagenous phosphoprotein. Arch Oral BioI 2002; 47(11): 757-62.

7. Ohtani S, Yamamoto T, Sugimoto H, Sashima M, Satoh M. Age-related changes in the D-aspartic acid content of the teeth of the senescenceaccelerated mouse. Arch Oral BioI. 2000 Jan; 45(1): 13-8.

8. Mornstad, H., Pfeiffer, H., Teivens, A. Estimation of dental age using HPLC-technique to determine the degree of aspartic acid racemization. J. For Sci. 1994; 39(6): 1425-31.

9. Waite ER, Collins MJ, Ritz-Timme S, Schutz HW, Cattaneo C, Borrman HI. A review of the methodological aspects of aspartic acid racemization analysis for use in forensic science. Forensic Sci Int. 1999 Jul 26; 103(2): 113-24.

10. Cloos PA and Jensen AI. age-related dephosphorylation of proteins in dentine: a biological tool for assessment of protein age. Biogerontology 2000; 1(4): 341-56. 